\title{
Exosome-encapsulated microRNA-223-3p as a minimally invasive biomarker for the early detection of invasive breast cancer
}

\author{
MIO YOSHIKAWA, HISAE IINUMA, YASUKO UMEMOTO, TAKAKO YANAGISAWA, \\ AKIKO MATSUMOTO and HIROMITSU JINNO
}

Department of Surgery, Teikyo University School of Medicine, Tokyo 173-0003, Japan

Received December 22,2017; Accepted March 16, 2018

DOI: $10.3892 / \mathrm{ol} .2018 .8457$

\begin{abstract}
Patients diagnosed preoperatively with ductal carcinoma in situ (DCIS) breast cancer have the potential to develop invasive ductal carcinoma (IDC). The present study investigated the usefulness of exosome-encapsulated microRNA-223-3p (miR-223-3p) as a biomarker for detecting IDC in patients initially diagnosed with DCIS by biopsy. The potential association between miR-223-3p and clinicopathological characteristics was examined in patients with breast cancer. Exosomes of 185 patients with breast cancer were separated from plasma by ultracentrifugation. Initially a microRNA (miRNA) microarray was examined to reveal the invasion specific miRNAs using exosomes collected from 6 patients with breast cancer, including 3 DCIS patients, 3 IDC patients and 3 healthy controls. In the miR microarray analysis the miR-223-3p levels of IDC patients demonstrated the highest fold-change compared with those in the DCIS patients and healthy controls. The potential of miR-223-3p for cell proliferation and cell invasion were examined in vitro using MCF7 cells transfected with the miR-223-3p gene. MCF7 cells transfected with the miR-223-3p gene significantly promoted cell proliferation and cell invasive ability $(\mathrm{P}<0.05)$. The plasma exosomal miR-223-3p levels of the other 179 patients with breast cancer and 20 healthy controls were measured using TaqMan miR assays. The exosomal miR-223-3p levels of the patients with breast cancer were significantly increased compared with the healthy controls $(\mathrm{P}<0.01)$. A statistically significant association was observed between the exosomal miR-223-3p levels
\end{abstract}

Correspondence to: Dr Hisae Iinuma, Department of Surgery, Teikyo University School of Medicine, 2-11-1 Kaga, Itabashi, Tokyo 173-0003, Japan

E-mail: iinuma@med.teikyo-u.ac.jp

Abbreviations: DCIS, ductal carcinoma in situ; RT-qPCR, reverse transcription-quantitative polymerase chain reaction; IDC, invasive ductal carcinoma; HER2, human epidermal growth factor receptor type 2; NG, nuclear grade; ER, estrogen receptor; PgR, progesterone receptor

Key words: breast cancer, biomarker, exosome, microRNA-223-3p, ductal carcinoma in situ, detecting invasive ductal carcinoma and histological type, $\mathrm{pT}$ stage, $\mathrm{pN}$ stage, pathological stage, lymphatic invasion and nuclear grade $(\mathrm{P}<0.05)$. The exosomal miR-223-3p levels of IDC patients (stage I) and upstaged IDC patients (stage I) were significantly higher compared with the DCIS patients $(\mathrm{P}<0.05)$. These results suggest that exosomal miR-223-3p may be a useful preoperative biomarker to identify the invasive lesions of DCIS patients diagnosed by biopsy. In addition, plasma exosome-encapsulated miR-223-3p level was significantly associated with the malignancy of breast cancer.

\section{Introduction}

Breast cancer is one of the most common forms of cancer among Japanese women, and about 70,000 women were diagnosed with breast cancer in 2015 (1). After the advent of screening mammography, there was an increase in the reported number of small invasive tumors (measuring $<2 \mathrm{~cm}$ or in situ carcinomas) (2). Ductal carcinoma in situ (DCIS) is a heterogeneous disease that currently accounts for approximately $20 \%$ of all screening-detected breast cancers (3). This disease is characterized by a proliferation of neoplastic ductal epithelial cells that are confined to the basement membrane of the mammary ducts. However, lesions initially diagnosed as DCIS in needle biopsy are occasionally upstaged to invasive ductal carcinoma (IDC) after the final pathology report on the completely excised specimen. It has been reported that the IDC identification rates at final pathology in patients initially diagnosed with DCIS by core-needle biopsy or vacuum-assisted biopsy devices are between 8 and 38\% (4,5). Therefore, clarification of the invasive cancer in patients diagnosed with DCIS by needle biopsy is important in deciding treatment strategy. The ability to distinguish DCIS and IDC at an earlier stage, would enable earlier individualized treatment options.

MicroRNAs (miRNAs) are 18-25 nucleotides single stranded non-coding RNAs. They regulate gene expression at the transcriptional or post-transcriptional level, and can act as either tumor suppressor genes or oncogenes, depending on the roles of their target mRNAs (6). An increasing number of studies have shown aberrant expression profiles of miRNAs in breast cancer, and reported on the usefulness of miRNA for diagnosis of breast cancer (7). Recently, miRNAs have been identified in plasma and serum (plasma/serum) and are considered as minimally invasive liquid biomarkers for diagnosis, prognosis, and therapeutic outcome in various cancer 
patients, including the breast cancer (8). Furthermore, these circulating miRNAs have been identified in the exosome of plasma/serum in a stable form, which is protected from endogenous RNase activity (9). Exosomes are 50-150 nm in diameter membrane-derived vesicles that are actively secreted from many cell types. They contain protein, lipids, mRNAs and miRNAs, and can transfer these components to other cells (10-12). In general, cancer cells secrete exosomes and cancer patients show high concentrations of exosomes in the blood. Therefore, exosomes which encapsulate intact miRNA could be potential biomarkers of malignancy of cancer (13-15). However, there is currently little available information regarding the relationship between the exosomal miRNA expression profiles and the pathological condition in patients with breast cancer. In particular, few reports have been published on the exosome miRNA characteristics of DICS and IDC patients with breast cancer.

In this study, we examined the usefulness of plasma exosomal miRNAs in the selection of patients with invasive lesions in DICS patients diagnosed using needle biopsy. Furthermore, we aimed to clarify the relationship between this invasion-specific exosomal miRNA and the clinicopathological factors of breast cancer.

\section{Patients and methods}

Study design and clinical samples. In this study, 185 breast cancer patients were included. Clinical samples were obtained from breast cancer patients who underwent surgery between June 2014 and May 2017 at Teikyo University School of Medicine (Tokyo, Japan). For a healthy control, 20 healthy volunteers were included. Peripheral blood and plasma samples were collected before the start of treatment. This study protocol conformed to the guidelines of the ethics committee, and was approved by the review board of the Teikyo University (09-081-3). Written informed consent was obtained from all the patients.

First, we selected the plasma exosomal miRNA which can distinguish between DCIS and IDC of breast cancer by the miRNA array using exosomes collected from DCIS patients $(n=3)$, IDC patients $(n=3)$ with stage I and healthy controls $(n=3)$. Next, we examined the potential of selected miRNA for cell proliferation and cell invasion using the MCF7 cell lines in vitro. Third, we clarified the usefulness of selected plasma exosomal miRNA as biomarker for distinguishing early IDC from another 43 patients diagnosed with DCIS by preoperative needle biopsy. Finally, we examined the relationship between the selected miRNA and clinicopathological characteristics using the exosomes collected from 179 patients with breast cancer.

Cell culture. The human breast cancer cell line MCF7 was obtained from the Cell Resource Center for Biomedical Research Institute of Development, Aging and Cancer, Tohoku University. The Cell line was maintained in RPMI-1640 supplemented with $10 \%$ fetal bovine serum (FBS) at $37^{\circ} \mathrm{C}$ in a $5 \%$ humidified $\mathrm{CO}_{2}$ atmosphere.

Transfection and establishment of pre-miR-223-3p-stably transfected MCF7 cell line. The backbone plasmid pcDNA6.2-GW/EmGFP-miR was obtained from the Block-iT Pol II miR RNAi Expression Vector kit (Thermo Fisher Scientific, Inc., Waltham, MA, USA). The plasmids pcDNA6.2-GW/EmGFP-pre-miR-223-3p (pCMV-pre-miR-223-3p) containing pre-miR-223-3p, and pcDNA6.2-GW/EmGFP-miR-neg (pCMV-N) containing an unrelated insert were constructed according to the manual for the Block-iT Pol II miR RNAi Expression Vector kit, as described previously (16). The sequence of mature miR-223-3p was UGUCAGUUUGUCAAAUAC CCCA (has-miR-223-3p). The pCMV-pre-miR-223-3p and pCMV-miR-neg were transfected into the MCF7 cell line using the lipofectamine 3000 (Life Technologies, Inc, Tokyo, Japan) according to the manufacturer's instructions. Then stably transfected cells expressing mature miR-223-3p were selected with G418 treatment followed by sorting with GFP by MACS. A pCMV-miR-neg-transfected clone of the cell line was used for the control. Expressions of miR-223-3p of pCMV-pre-miR-223-3p or wpCMV-miR-neg-transfected MCF7 cells were measured by reverse transcription-quantitative polymerase chain reaction (RT-qPCR) system (StepOne; Thermo Fisher Scientific, Inc.) and Digital PCR system (Quant Studio 3D Digital PCR System; Thermo Fisher Scientific, Inc.).

Cell proliferation assay. Cell proliferation was evaluated by performing MTT assays using a Cell Proliferation kit 1 (Roche Applied Science, Penzberg, Germany) according to the manufacturer's instructions. miR-223-3p-transfected cells and mock-transfected cells were seeded at 3,000 cells per well in triplicate 96-wells in $100 \mu \mathrm{l}$ medium. The coloring reaction was quantitated using a plate reader, at $570 \mathrm{~nm}$ with a reference filter of $650 \mathrm{~nm}$. Each independent experiment was performed in triplicate.

Cell invasion assay. Cell invasion capacities were assessed using the BD BioCoat Tumor Invasion System, 24 Multiwell (BD Bioscience) according to the manufacturer's instructions as described previously (16). In brief, miR-223-3p-transfected cells and mock-transfected cells $\left(10^{5}\right.$ cells/well $)$ were placed in the upper chamber, and the lower chamber was filled with $750 \mu \mathrm{l}$ of RPMI-1640 with $10 \%$ FBS and incubated in a humidified atmosphere $\left(37^{\circ} \mathrm{C}\right.$ and $\left.5 \% \mathrm{CO}_{2}\right)$. After $48 \mathrm{~h}$ incubation, the upper chamber was transferred into a second 24-well plate, with each well containing $500 \mu \mathrm{l}$ of $4 \mu \mathrm{g} / \mathrm{ml}$ calcein AM solution. The plates were incubated for an additional $1 \mathrm{~h}$ $\left(37^{\circ} \mathrm{C}\right.$ and $\left.5 \% \mathrm{CO}_{2}\right)$. Invasive cells that advanced through the membrane were evaluated in a fluorescence plate reader at excitation/emission wavelengths of 485/535 nm. Each independent experiment was performed in triplicate.

Purification of exosome from plasma. Peripheral blood was separated by centrifugation at $2,000 \mathrm{x}$ g for $15 \mathrm{~min}$ at $4^{\circ} \mathrm{C}$ in order to collect plasma. Plasma of $1.0 \mathrm{ml}$ was used for microarray analysis and reverse transcription-quantitative PCR (RT-qPCR). Exosomes of plasma were separated by ultracentrifugation at $15,000 \mathrm{x}$ for $70 \mathrm{~min}$. at $4^{\circ} \mathrm{C}$, and the pellets were washed with phosphate-buffered saline (PBS) and prepared for microarray and RT-qPCR analysis.

Confirmation of exosome by electron microscopic image. Isolated exosomes were confirmed by transmission electron 
microscopy. Isolated exosomes were dissolved in PBS buffer, and a drop of the suspension was placed on a sheet. A carbon-coated copper grid was floated on the drop for 10s. Then the grid was removed and excess liquid drained by filter paper. The grid was put in contact with a drop of $2 \%$ uranyl acetate of phosphotungstic acid for $5 \mathrm{~s}$. After remove of the excess liquid, the grid was allowed to dry for several min and was then observed using electron microscope (HITACHI H-7600; Hitachi, Ltd., Tokyo, Japan).

Total RNA isolation from exosomes and tissues. Total RNAs (including the miRNA) of exosomes were isolated using the miRNeasy serum/plasma kit (Qiagen, Inc., Valencia, CA, USA), and total RNAs (including the miRNA) of tissues were extracted using the miRNeasy Mini kit (Qiagen, Inc.). Subsequent procedure was performed according to the manufacturer's protocol described previously (17). Using an Agilent 2100 Bioanalyzer, quality of extracted RNA was examined (Agilent Technologies, Inc., Santa Clara, CA, USA).

miRNA microarray analysis. Exosomal miRNA expression profiles were examined using a 3D-Gene Human miRNA Oligo chips ver.21 (Toray Industries Inc., Tokyo, Japan), according to the manufacturer's protocol. Fluorescence signals were scanned and analyzed using the 3D-Gene Scanner (Toray). The number of genes mounted in this chip was 2,565 . The raw data for each spot were normalized to the mean intensity of background signals determined by all blank signal intensities at a $95 \%$ confidence intervals. Valid measurements were considered those in which the signal intensity of both duplicate spots was $>2$ SD of the background signal intensity.

RT-qPCR for miRNA of exosomes and tumor tissues. miRNA expressions of plasma exosomes and tissues were assayed using RT-qPCR as described previously (18). All primers, reagents and assay kits for TaqMan RT-qPCR assays were purchased from ThermoFisher Scientific, Inc. Complementary DNA (cDNA) of exosomes or tissues samples was synthesized from total RNA using TaqMan MicroRNA primers specific for miR-223-3p (002295) and TaqMan MicroRNA RT kit. miR-16a (000391) was used as an internal control for exosomal samples, and RNU-6B (001093) was used as an internal control for tissue samples. RT-qPCR was performed using TaqMan Universal PCR Master Mix and StepOne, following the manufacturer's protocol. Each sample was analyzed in triplicate. Relative quantification of miRNA expression was calculated using the $2^{-\Delta \Delta C q}$ method as described previously (18).

RT-qPCR for EPB41L3 mRNA of tumor tissues. Total RNAs were extracted by miRNeasy Mini kit (Qiagen, Inc.) and cDNA synthesis was performed using random hexamer primers and SuperScript II reverse transcriptase according to the manufacturer's protocol (Thermo Fisher Scientific. Inc.). The amplifications of EPB41L3 mRNA (Hs00202360) and GAPDH mRNA (Hs03929097) were performed using the TaqMan primer and probe set (Thermo Fisher Scientific, Inc.) and the RT-qPCR of these mRNAs was measured using StepOne. GAPDH mRNA was used as an internal control for this assay. All the experiments were performed in triplicate.

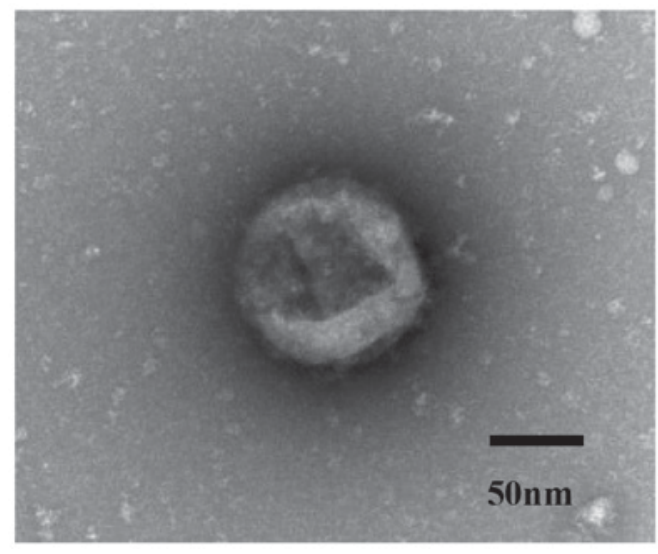

Figure 1. Plasma exosome image by transmission electron microscope.

The expression levels of EPB41L3 mRNA were normalized to GAPDH mRNA expression levels.

Statistical analysis. The data were expressed as mean \pm standard deviation. The relationships between microRNA expression and clinicopathological factors were analyzed using the Student's t-test, the chi-squire test and one-way analysis of variance (ANOVA). Tukey-HSD was used as post hoc test after one-way ANOVA. Univariate analysis was examined for each factor, and multivariate analysis was performed for factors that showed significance in univariate analysis. All $\mathrm{P}$-values are two-sided, and $\mathrm{P}<0.05$ was considered to indicate a statistically significant difference. Statistical analyses were performed using the JMP 9.0 software (SAS Institute, Inc., Cary, NC, USA).

\section{Results}

Identification of exosome in plasma. To confirm the isolation of exosome, we examined the ultracentrifugation samples from the plasma of breast cancer patients using transmission electron microscopy. We captured images of micro vesicles with diameters of about $100 \mathrm{~nm}$ (Fig. 1).

Exosomal miRNA profile of DCIS and IDC breast cancer patients. To clarify the exosomal miRNA useful for discriminating between the DCIS and IDC of the breast cancer patients, we examined the miRNA microarray analyses of samples from 3 DCIS patients, 3 IDC patients with stage I and 3 healthy controls. The clinical characteristics of these patients are shown in Table I. The average age of DCIS and IDC patients was 66 years (range, 49-78) and 59 years (range, 38-75), respectively. The degree of vascular invasion, lymph node metastasis, nuclear grade (NG), estrogen receptor (ER) and progesterone receptor $(\mathrm{PgR})$ were the same in DCIS and IDC patients. The average value of Ki67 labeling index (Ki67) of IDC patients is higher than that of DCIS patients; however it was not a significant difference. Table II shows the 5 most highly upregulated exosomal miRNAs among 2565 miRNAs in these breast cancer patients. In the exosomal samples, the miR-223-3p (MIMAT0000280) of IDC patients showed the highest fold-change (3.45 times) as compared with that of the healthy controls. And the miR-223-3p of IDC patients 
Table I. Characteristics of patients used for miRNA microarray analyses.

\begin{tabular}{|c|c|c|}
\hline Variable & DCIS $(n=3)$ & IDC $(n=3)$ \\
\hline Age, year (range) & $66(49-78)$ & $59(38-75)$ \\
\hline Tumor size, cm (range) & 0 & $1.8(0.3-2.0)$ \\
\hline \multicolumn{3}{|l|}{ Pathological stage } \\
\hline 0 & 3 & 0 \\
\hline 1 & 0 & 3 \\
\hline \multicolumn{3}{|l|}{ Vascular invasion } \\
\hline$(-)$ & 3 & 3 \\
\hline$(+)$ & 0 & 0 \\
\hline \multicolumn{3}{|l|}{ Lymph node metastasis } \\
\hline$(-)$ & 3 & 3 \\
\hline$(+)$ & 0 & 0 \\
\hline \multicolumn{3}{|l|}{ NG } \\
\hline 1 & 2 & 2 \\
\hline 2 & 1 & 1 \\
\hline 3 & 0 & 0 \\
\hline \multicolumn{3}{|l|}{ ER } \\
\hline$(-)$ & 1 & 1 \\
\hline$(+)$ & 2 & 2 \\
\hline \multicolumn{3}{|l|}{$\operatorname{PgR}$} \\
\hline$(-)$ & 1 & 1 \\
\hline$(+)$ & 2 & 2 \\
\hline HER 2 & & 3 \\
\hline$(-)$ & $-{ }^{\mathrm{a}}$ & 3 \\
\hline$(+)$ & $-a^{a}$ & 0 \\
\hline Ki67, \% (range) & $9(5-15)$ & $14(7-20)$ \\
\hline
\end{tabular}

${ }^{\text {aHER} 2 ~ d e t e r m i n a t i o n s ~ w e r e ~ n o t ~ p e r f o r m e d . ~ A g e, ~ t u m o r ~ s i z e ~ a n d ~ K i 67 ~}$ are average values. DCIS, ductal carcinoma in situ; IDC, invasive ductal carcinoma; NG, nuclear grade; ER, estrogen receptor; PgR, progesterone receptor.

also showed a high fold-change (2.85 times) as compared with that of the DCIS patients. We selected miR-223-3p as a potential marker for diagnosis of early invasion in breast cancer patients.

Expression of miR-223-3p in miR-223-3p-transfected MCF7 cells. The miR-223-3p gene expressions of pCMV-pre-miR-223-3p transfected (miR-223-3p-transfected) MCF7 cells and pCMV-N transfected (mock-transfected) MCF7 cells were measured by RT-qPCR and Digital PCR. In the RT-qPCR assay, a significant amplification of miR-223-3p was shown in the miR-223-3p-trasnfected MCF7 cells as compared with that of the mock-transfected cells (Fig. 2A). In the digital PCR assay, the copy number of miR-223-3p of the miR-223-3p-transfected MCF7 cells was significantly higher than that of the mock-transfected MCF7 cells (Fig. 2B).

Cell proliferation and cell invasion potentials. To examine the proliferation potential of the MCF7 cells transfected with
miR-223-3p, MTT assays were performed. As shown in Fig. 3A, MCF7 cells transfected with miR-223-3p showed a significant increase of proliferation compared to that of mock-transfected MCF7 cells $(\mathrm{P}<0.05)$. To evaluate the cell invasion potential of the MCF7 cells transfected with miR-223-3p, invasion assays were performed. As presented in Fig. 3B, the invasive ability of MCF7 cells transfected with miR-223-3p was significantly increased compared to that of mock-transfected MCF7 cells $(\mathrm{P}<0.05)$.

Expression of miR-223-3p in the plasma exosomes and breast cancer tissues. We examined the correlation between the exosomal miR-223-3p levels and the miR-223-3p expressions in primary tumor tissues collected from same breast cancer patients. This study consisted of 40 breast cancer patients, including DCIS patients $(n=5)$ and stage I patients $(n=15)$, stage II patients $(n=15)$ and stage III patients $(n=5)$. As shown in Fig. 4, a positive significant correlation was demonstrated between them $(\mathrm{P}<0.01)$. Fig. 5 shows a comparison of exosomal miR-223-3p levels between the breast cancer patients and the healthy controls. The exosomal miR-223-3p levels of the breast cancer patients were significantly higher than those of the healthy controls $(\mathrm{P}<0.01)$. Furthermore, exosomal miR-223-3p levels increased according to the advance in stage (stage 0 vs. stage II and stage III: $\mathrm{P}<0.01$; stage I vs. stage 0 , stage II and stage III: $\mathrm{P}<0.05)$.

Relationship between clinicopathological factors and exosomal miR-223-3p levels. To evaluate the correlation between the miR-223-3p expression levels and the clinicopathological characteristics, 179 breast cancer patients were divided into two groups (high and low expression). The cut-off level of miR-223-3p was determined as the median of the relative quantity (median=2.80). The average age was 58 years (range, 33-88). All patients were female. As shown in Table III, a statistically significant association was observed between high miR-223-3p expression and histological type, $\mathrm{pT}$ stage, pN stage, pathological stage, lymphatic invasion and NG $(\mathrm{P}<0.05)$.

Comparison of exosomal miR-223-3p levels of DCIS, upstaged IDC and IDC patients. At the time of diagnosis by biopsy before surgery, there were 43 DCIS patients and 76 IDC patients with stage I. Of the 43 DCIS patients diagnosed by biopsy, 18 patients (41.9\%) upstaged to IDC patients with stage I in the final pathological diagnosis by completely excised specimen. Fig. 6 shows a comparison of the plasma exosomal miR-223-3p levels of DCIS patients diagnosed by completely excised specimen $(n=25)$, upstaged IDC patients with stage I $(n=18)$, and IDC patients with stage I $(n=76)$. In our study, miR-223-3p levels of IDC patients and upstaged IDC patients were significantly higher than those of DCIS patients $(\mathrm{P}<0.05)$.

Correlation between miR-223-3p levels and target gene. Using the target gene prediction program TargetScan and PicTar, we selected EPB41L3 as one of the direct target genes of miR-223-3p (data not shown). This study consisted of 40 breast cancer patients, including DCIS patients $(n=5)$ and stage I patients $(n=15)$, stage II patients $(n=15)$ and stage III patients $(n=5)$. The correlation between the 
Table II. Five most highly upregulated miRNAs based on the miRNA microarray.

Fold-changes

\begin{tabular}{lllll} 
Rank & miR healthy & MirBase no. & IDC vs. controls & IDC vs. DCIS \\
\hline 1 & miR-223-3p & MIMAT 0000280 & 3.45 & 2.85 \\
2 & miR-130a-3p & MIMAT 0000278 & 3.11 & 2.03 \\
3 & miR-191-5p & MIMAT 0000440 & 3.25 & 2.14 \\
4 & miR-146a & MIMAT 0000092 & 2.93 & 2.08 \\
5 & miR-221-3p & MIMAT 0000278 & 2.73 & 2.24 \\
\hline
\end{tabular}

IDC, invasive ductal carcinoma; DCIS, ductal carcinoma in situ; miR, microRNA.

A

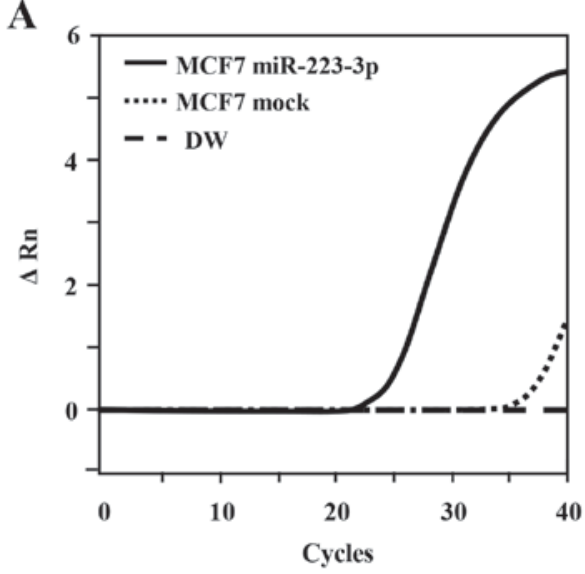

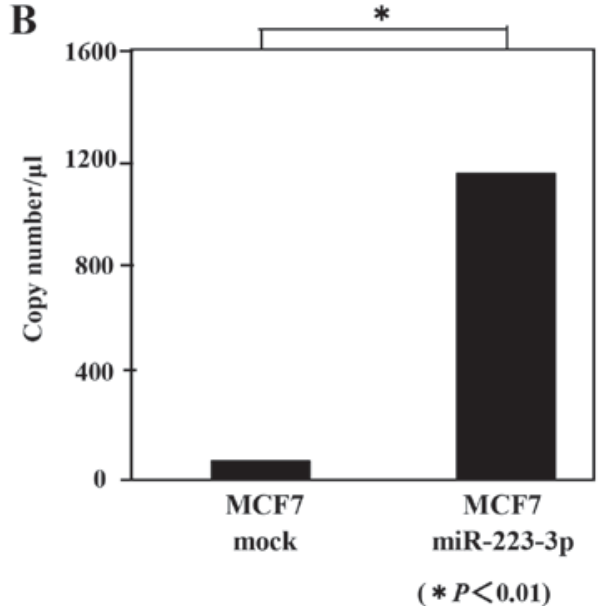

Figure 2. Expression of miR-223-3p gene in MCF7 cells transfected with miR-223-3p. (A) Expression of the miR-223-3p gene in miR-223-3p-transfected MCF7 cells and mock-transfected MCF7 cells were examined by reverse transcription-quantitative PCR. (B) Expression of the miR-223-3p gene in MCF miR-223 and MCF7 mock were examined by digital PCR. "P<0.01. DW, distilled water; PCR, polymerase chain reaction; miR, microRNA.

miR-223-3p and the EPB41L3 mRNA levels in the breast cancer tissues was examined. As shown in Fig. 7, a significant inverse correlation was shown between miR-223-3p and the EPB41L3 mRNA expressions in the breast cancer tissues $(\gamma=0.70, P<0.01)$.

\section{Discussion}

In the present study, we demonstrated that miR-223-3p has the potential to enhance cell proliferation and invasion of breast cancer cells. In breast cancer patients, exosomal miR-223-3p levels showed a significant relationship with histological type, pT stage, $\mathrm{pN}$ stage, pathological stage, lymphatic invasion and NG. Furthermore, we clarified that the plasma exosomal miR-223-3p levels of patients upstaged from DCIS to IDC were significantly higher than those of the DCIS patients.

A number of recent studies have supported a model of breast cancer development in which atypical ductal hyperplasia evolves into DCIS. It is known that disruption of the myoepithelial layer and the basement membrane in DCIS progresses to IDC (19). DCIS is considered to be a direct precursor to IDC. Some studies have reported that lesions initially diagnosed as DCIS by core-needle biopsy or vacuum-assisted biopsy are occasionally upstaged to IDC after the final diagnosis of the completely excised specimen. One study $(n=336)$ has reported that this underestimation rate was $33.6 \%$ and another meta-analysis study $(\mathrm{n}=7350)$ showed an underestimation rate was $25.9 \%(5,20)$. In our study, $41.9 \%$ (18/43) of DCIS patients upstage to IDC after the final diagnosis of the completely excised specimen. By accurate prediction of DCIS and IDC before surgery, more appropriate individualized treatment for these patients can be planned at an earlier time. Early diagnosis of IDC may lead to more effective treatment. This underestimation is mostly occurring due to inherent limitations in biopsy sampling techniques, whereby a small invasive lesion may escape detection within the large area of the intraductal lesion. Previous studies have focused on clinico-histological or clinico-radiological predictors for underestimation of invasion in biopsy samples $(21,22)$. Meta-analysis has indicated that the preoperative variables most significantly associated with underestimation include the biopsy device and guidance method, size, grade, mammographic features, and palpability (5). However, the relative importance of these various predictors of invasive breast cancer and their impact on patient outcome has not been clearly established. In our study, these clinical factors did not show a relationship with the invasion of breast cancer (23). Therefore, a novel biomarker to distinguish the invasion lesion hidden in the DCIS patients is necessary. 
A

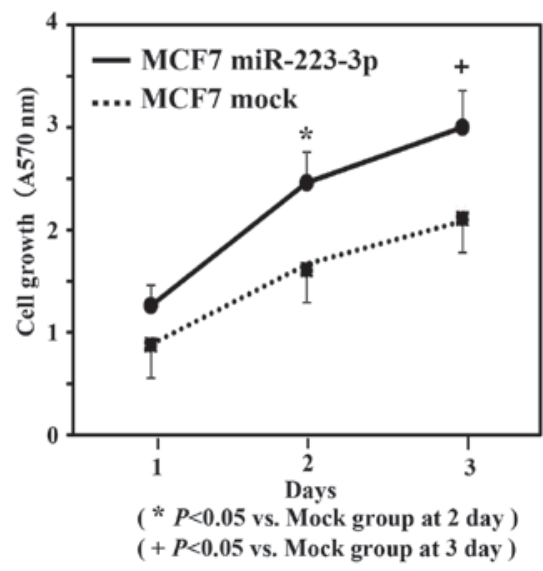

B

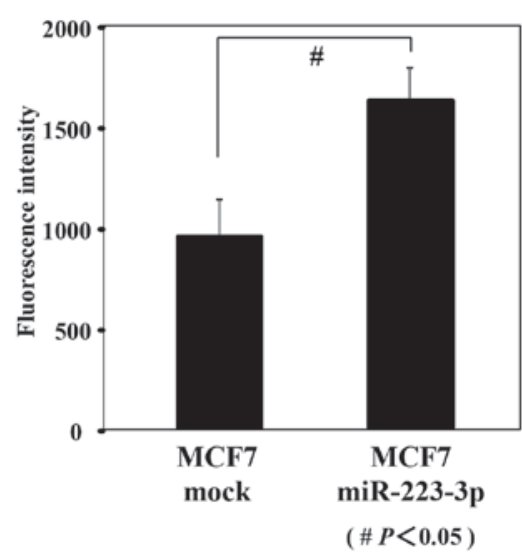

Figure 3. Potential of cell proliferation and cell invasion of MCF7 cells transfected with miR-223-3p. (A) Cell proliferation assays were examined using the MCF7 miR-223-3p and MCF7 mock. (B) Cell invasion assays were examined using the MCF7 miR-223-3p and MCF7 mock. ${ }^{*} \mathrm{P}<0.05$, ${ }^{*} \mathrm{P}<0.05$ and ${ }^{+\mathrm{P}}<0.05$. miR, microRNA.

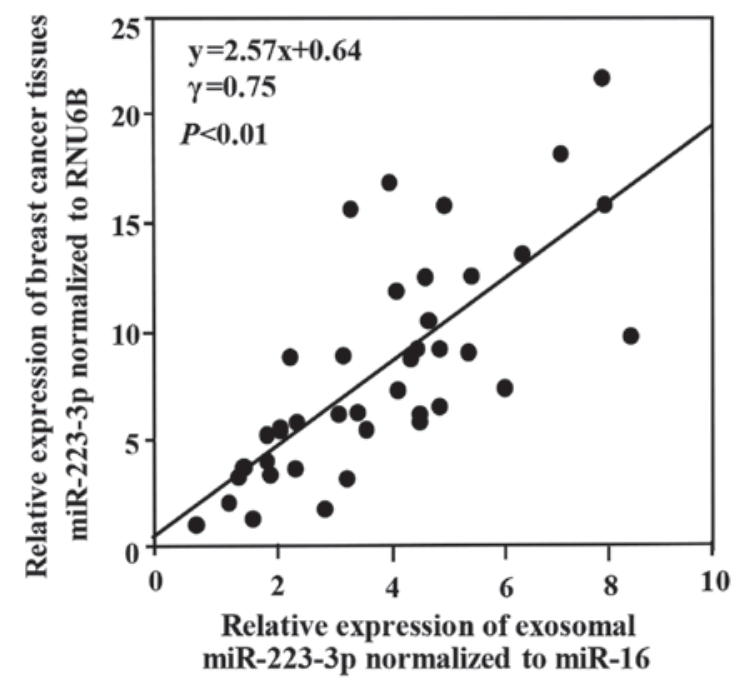

Figure 4. Correlation between miR-223-3p levels in plasma exosomes and breast cancer tissues. This study consisted of 40 patients with breast cancer, including DCIS $(n=5)$, stage I $(n=15)$, stage II $(n=15)$ and stage III patients $(n=5)$. The pair samples of plasma and tissue were collected from the same patients. DCIS, ductal carcinoma in situ; miR, microRNA.

Recently, some researchers have reported on the potential of diagnostic biomarkers of circulating plasma/serum miRNA in breast cancer patients using various kinds of miRNA $(8,24)$. In particular, exosome of plasma/serum is attractive because the miRNAs are preserved in a stable form in the exosome. However, until now, there are few reports that have compared IDC and DCIS with miRNA microarrays using exosome samples. First, we compared the profile of miRNA in DCIS patients, IDC patients and healthy controls, using the miRNA microarray. In our study, exosomal miR-223-3p in IDC patients revealed the highest fold-change compared with the DCIS patients and healthy controls. Therefore we selected miR-223-3p as on invasion specific biomarker for breast cancer patients.

Next, we examined the potential of miR-223-3p against proliferation and invasion of breast cancer cells. Our results demonstrated that breast cancer MCF7 cells transfected

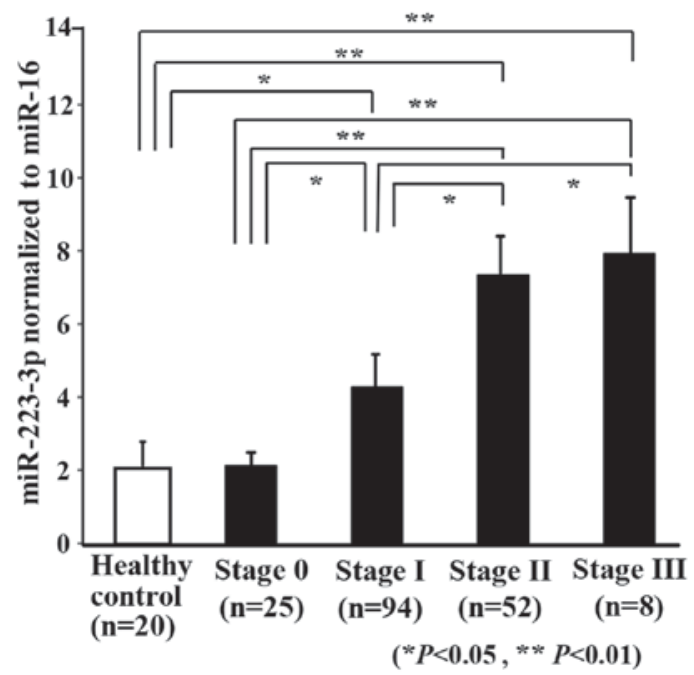

Figure 5. Comparison of exosomal miR-223-3p levels in healthy control and patients with breast cancer at each tumor stage. Exosomal miR-223-3p levels of healthy controls $(n=20)$ and patients with breast cancer at stage $0(n=25)$, stage I $(n=94)$, stage II $(n=52)$ and stage III $(n=8)$ were measured by reverse transcription-quantitative polymerase chain reaction. ${ }^{*} \mathrm{P}<0.05,{ }^{* *} \mathrm{P}<0.01$. miR, microRNA.

with miR-223-3p significantly increased proliferation and invasion. As for the function of miR-223, Huang et al have revealed that miR-223 increase proliferation and promote invasion of lung cancer A549 cells via activation of the NF- $\mathrm{KB}$ signaling pathway (25). Also of interest is a reported by Li et al that miR-223 is overexpressed in metastatic gastric cancer cells and promotes gastric cancer invasion and metastasis (26). These manuscripts support our results. In contrast, Pinatel et al reported that a suppressive role of miR-223 in the migration of breast cancer (27). The exact reasons for the discrepancy in these results remain unknown and wait further study. Since this manuscript does not separately describe miR-223, miR-223-3p and miR-223-5p, further analysis in which patients are divided into miR-223-3p and miR-223-5p may be necessary.

The clinical characteristics of exosomal miR-223-3p in breast cancer patients have not been fully reported. In this 
Table III. Association between clinicopathological factors and exosomal miR-223-3p levels.

\begin{tabular}{|c|c|c|c|c|}
\hline Variable & No. of patients $n=179$ & High $n=84(\%)$ & Low $n=95(\%)$ & P-value \\
\hline Histological type & & & & $<0.001$ \\
\hline DCIS & 25 & $3(3.6)$ & $22(23.2)$ & \\
\hline IDC & 154 & $81(96.4)$ & $73(76.8)$ & \\
\hline pT Stage & & & & $<0.001$ \\
\hline 0 & 27 & $5(6.0)$ & $22(23.2)$ & \\
\hline 1 & 119 & $56(66.6)$ & $63(66.3)$ & \\
\hline 2 & 33 & $23(27.4)$ & $10(10.5)$ & \\
\hline pN Stage & & & & 0.020 \\
\hline 0 & 140 & $58(69.1)$ & $82(86.3)$ & \\
\hline 1 & 31 & $21(25.0)$ & $10(10.5)$ & \\
\hline 2 & 8 & $5(5.9)$ & $3(3.2)$ & \\
\hline Pathological stage & & & & $<0.001$ \\
\hline 0 & 25 & $3(3.6)$ & $22(23.2)$ & \\
\hline I & 94 & $41(48.8)$ & $53(55.8)$ & \\
\hline II & 52 & $34(40.5)$ & $18(18.9)$ & \\
\hline III & 8 & $6(7.1)$ & $2(2.1)$ & \\
\hline Lymphatic invasion & & & & 0.048 \\
\hline$(-)$ & 107 & $44(52.4)$ & $63(66.3)$ & \\
\hline$(+)$ & 72 & $40(47.6)$ & $32(33.7)$ & \\
\hline Vascular invasion & & & & 0.559 \\
\hline$(-)$ & 119 & $54(64.3)$ & $65(68.4)$ & \\
\hline$(+)$ & 60 & $30(35.7)$ & $30(31.6)$ & \\
\hline NG & & & & 0.020 \\
\hline 1 & 122 & $61(72.6)$ & $61(64.2)$ & \\
\hline 2 & 24 & $5(6.0)$ & $19(20.0)$ & \\
\hline 3 & 33 & $18(21.4)$ & $15(15.8)$ & \\
\hline ER & & & & 0.510 \\
\hline$(-)$ & 20 & $8(9.5)$ & $12(12.6)$ & \\
\hline$(+)$ & 159 & $76(90.5)$ & $83(87.4)$ & \\
\hline $\mathrm{PgR}$ & & & & 0.736 \\
\hline$(-)$ & 49 & $24(28.6)$ & $25(26.3)$ & \\
\hline$(+)$ & 130 & $60(71.4)$ & $70(73.7)$ & \\
\hline $\mathrm{HER}^{\mathrm{a}}{ }^{\mathrm{a}}$ & & & & 0.072 \\
\hline$(-)$ & 143 & $76(97.4)$ & $67(90.5)$ & \\
\hline$(+)$ & 9 & $2(2.6)$ & $7(9.5)$ & \\
\hline Ki67 & & & & 0.800 \\
\hline$\geq 15 \%$ & 39 & $19(22.6)$ & $20(21.1)$ & \\
\hline$<15 \%$ & 140 & $65(77.4)$ & 75 (78.9) & \\
\hline Intrinsic subtype & & & & 0.228 \\
\hline Luminal A & 108 & $61(78.2)$ & $47(63.5)$ & \\
\hline Luminal B & 28 & $11(14.1)$ & $17(23.0)$ & \\
\hline HER2 enriched ${ }^{a}$ & 4 & $1(1.3)$ & $3(4.0)$ & \\
\hline Triple negative & 12 & $5(6.4)$ & $7(9.5)$ & \\
\hline
\end{tabular}

${ }^{a}$ HER 2 determinations of 27 patients were not performed. The median level of miR-223-3p was used as the cut-off value for high and low expression. Bold print indicates $\mathrm{P}<0.05$. DCIS, ductal carcinoma in situ; IDC, invasive ductal carcinoma; NG, nuclear grade; ER, estrogen receptor; PgR, progesterone receptor.

study, we clarified that exosomal miR-223-3p levels of breast cancer were significantly higher than those found in healthy controls. In addition, we found a significant association between exosomal miR-223-3p levels and miR-223-3p expression in 


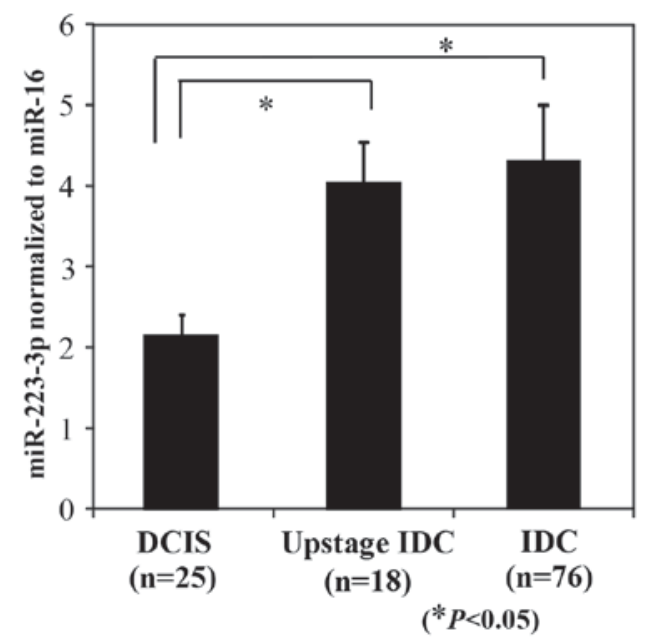

Figure 6. Comparison of exosomal miR-223-3p levels between DCIS patients, upstage IDC patients and IDC patients. Exosomal miR-223-3p levels of DCIS patients (Stage 0), upstaged IDC patients (Stage I) and IDC patients (Stage I) were measured by reverse transcription-quantitative polymerase chain reaction. DCIS patients who were initially diagnosed by a needle biopsy prior to surgery were re-diagnosed following the operation using the completely excised specimen. Patients upstaged to IDC from DCIS on the final pathological report were described as upstage IDC patients. DCIS, ductal carcinoma in situ; IDC, invasive ductal carcinoma; miR, microRNA.

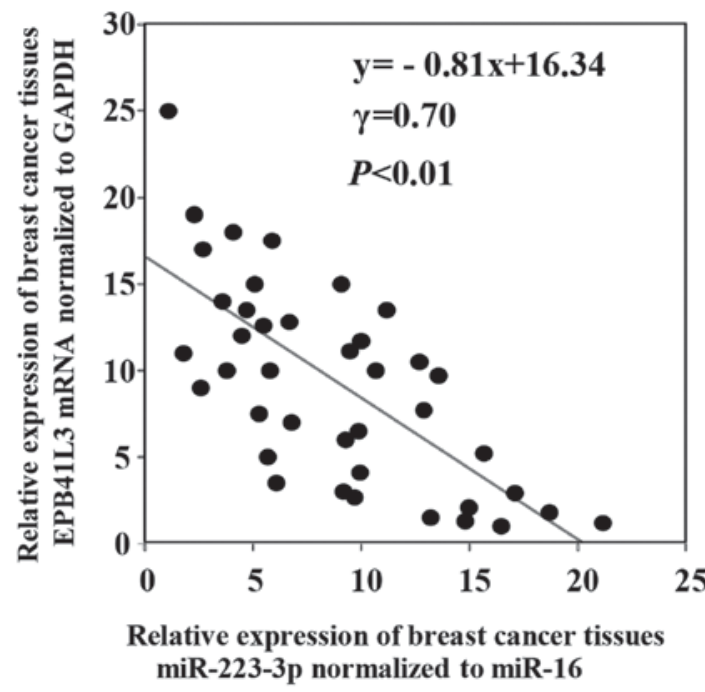

Figure 7. Correlation between miR-223-3p and EPB41L3 mRNA levels in breast cancer tissues. This study consisted of 40 breast cancer patients, including DCIS patients $(n=5)$ and stage I patients $(n=15)$, stage II patients $(\mathrm{n}=15)$ and stage III patients $(\mathrm{n}=5)$. DCIS, ductal carcinoma in situ; miR, microRNA.

primary breast cancer tissues collected from the same patients. These results suggest the possibility that tumor tissues may be the source of plasma exosomal miR-223-3p. Next, we investigated the relationship between exosome miR-223-3p levels and clinicopathological factors. We found that exosome miR-223-3p has relevance to the histological type, pT stage, $\mathrm{pN}$ stage, pathological stage, lymphatic invasion and NG. Furthermore, as a special point to note, we have revealed that exosomal miR-223-3p levels of IDC patients upstaged from DCIS by final diagnosis were significantly higher than those of the DCIS patients. To the best of our knowledge, this is the first study to show the potential of exosomal miR-223-3p in the detection of invasive lesions in DCIS patients diagnosed by biopsy. These results suggest the possibility that exosomal miR-223-3p may be a useful, less invasive biomarkers for the selection of IDC patients hidden among DCIS patients diagnosed by biopsy.

It is known that miRNAs downregulate gene expression by either inducing degradation of target mRNA or impairing their translation. By the data base analysis using the target gene prediction program TargetScan and PicTar, we selected EPB41L3 as a candidate target gene for miR223-3p. We found a significant inverse correlation between the miR-223-3p levels and the EPB41L3 mRNA levels. These results suggest that the expression of EPB41L3 mRNA may be negatively regulated by miR-223-3p. Li et al also reported that EPB41L3, which is a tumor suppressor gene, is a direct target gene for miR-223, and overexpression EPB41L3 can strongly inhibit migration and invasion of gastric cancer cells (26). As another target of miR-223, HAX-1 and STAT5A genes are reported $(27,28)$.

One of the limitations of our study is the small number of patients. Further research, conducted on a larger number of cases is required in order to validate the usefulness of miR-223-3p in the accurate detection of invasive lesions in DCIS patients diagnosed by biopsy. Furthermore, on inhibition test using the RNAi of miR-223-3p is also required in order to clarify the function of miR-223-3p in breast cancer cells. We are planning to investigate these points in our next study.

In summary, we have shown that miR-223-3p promotes the invasion of breast cancer cells, and exosomal miR-223-3p may be useful as a minimally invasive biomarker for the selection of patients with invasion from DSIC patients diagnosed by biopsy. We hope to be able to report on future developments in subsequent larger scale studies.

\section{Acknowledgements}

The authors would like to thank Miss J. Tamura for her technical assistance.

\section{Funding}

This study was supported by JSPS KAKENHI (grant no. JP17K10608).

\section{Availability of data and materials}

All data generated or analyzed during this study are included in this published article.

\section{Author's contributions}

HI designed the study. MY, HI and HJ wrote the manuscript. MY and HI performed the experiments. MY, AM, YU and TY collected the clinical data. MY, HI and HJ performed the statistical analysis. All authors read and approved the final manuscript.

\section{Ethics approval and consent to participate}

The study protocol conformed to the guidelines of the Teikyo University Ethics Committee and was approved by the review 
board of Teikyo University (approval no. 09-081-3). Written informed consent was obtained from all patients.

\section{Consent for publication}

Written informed consent was obtained from all patients for the publication of their data.

\section{Competing interests}

The authors declare that they have no competing interests.

\section{References}

1. The Editorial Board of the Cancer Statistics in Japan: Cancer Statistics in Japan 2015. Foundation for Promotion of Cancer Research. National Cancer Center, Tokyo, 2016. https://ganjoho.jp/ data/reg_stat/statistics/brochure/2015/cancer_statistics_2015.pdf. Accessed March 30, 2016.

2. Welch HG, Prorok PC, O'Malley AJ and Kramer BS: Breast-cancer tumor size, overdiagnosis and mammography screening effectiveness. N Engl J Med 375: 1438-1447, 2016.

3. Fentiman IS: The dilemma of in situ carcinoma of the breast. Int J Clin Pract 55: 680-683, 2001

4. Cox CE, Nguyen K, Gray RJ, Salud C, Ku NN, Dupont E, Hutson L, Peltz E, Whitehead G, Reintgen D and Cantor A: Importance of lymphatic mapping in ductal carcinoma in situ (DCIS): Why map DCIS? Am Surg 67: 513-519, 2001

5. Brennan ME, Turner RM, Ciatto S, Marinovich ML, French JR, Macaskill P and Houssami N: Ductal carcinoma in situ at core needle biopsy: Meta-analysis of underestimation and predictors of invasive breast cancer. Radiology 260: 119-128, 2011.

6. Grady WM and Tewai M: The next thing in prognostic molecular markers: microRNA signatures of cancer. Gut 59: 706-708, 2010

7. Graveel CR, Calderone HM, Westerhuis JJ, Winn ME and Sempere LF: Critical analysis of the potential for microRNA biomarkers in breast cancer management. Breast Cancer (Dove Med Press) 7: 59-79, 2015.

8. Shimomura A, Shiino S, Kawauchi J, Takizawa S, Sakamoto H, Matsuzaki J, Ono M, Takeshita F, Niida S, Shiizu C, et al: Novel combination of serum microRNA for detedting brest cnacer in the early stage. Cancer Sci 107: 326-334, 2016.

9. Ge Q, Zhou Y, Lu J, Bai Y, Xie X and Lu Z: miRNA in plasma exosome is stable under different storage conditions. Molecules 19: 1567-1575, 2014

10. Valadi H, Ekström K, Bossios A, Sjöstrand M, Lee JJ and Lötvall JO: Exosome-mediated transfer of mRNAs and microRNAs is a novel mechanism of genetic exchange between cells. Nat Cell Biol 9: 654-659, 2007.

11. Simons M and Raposo G: Exosomes-vesicular carriers for intercelluar communication. Curr Opin Cell Biol 21: 575-581, 2009.

12. Hannafon BN and Ding WQ: Intercelluar communication by exosome-derived microRNAs in cancer. Int J Mol Sci 14: $14240-14269,2013$.

13. Ogata-Kawata H, Izumiya M, Kurioka D, Honma Y, Yamada $Y$, Furuta K, Gunji T, Ohta H, Okamoto H, Sonoda H, et al: Circulating exosomal microRNAs as biomarkers of colon cancer. PLoS One 9: e92921, 2014.
14. Joyce DP, Kerin MJ and Dwyer RM: Exosome-encapsulated microRNAs as circulating biomarkers for breast cancer. Int J Cancer 139: 1443-1448, 2016.

15. Hannafon BN, Trigoso YD, Calloway CL, Zhao YD, Lum DH, Welm AL, Zhao ZJ, Blick KE, Dooley WC and Ding WQ: Plasma exosome microRNAs are indicative of breast cancer. Breast Cancer Res 18: 90, 2016.

16. Kurashige J, Hasegawa T, Niida A, Sugimachi K, Deng N, Mima K, Uchi R, Sawada G, Takahashi Y, Eguchi H, et al: Integrated molecular profiling of human gastric cancer identifies DDR2 as a potential regulator of peritoneal dissemination. Sci Rep 6: 22371, 2016.

17. Dejima $\mathrm{H}$, Iinuma $\mathrm{H}$, Kanaoka $\mathrm{R}$, Matsutani $\mathrm{N}$ and Kawamura $\mathrm{K}$ : Exosomal microRNA in plasma as a non-invasive biomarker for the recurrence of non-small cell lung cancer. Oncol Lett 13: 1256-1263, 2017.

18. Tsukamoto M, Iinuma H, Yagi T, Matsuda K and Hashiguchi Y: Circulating exosomal MicroRNA-21 as a biomarker in each tumor stage of colorectal cancer. Oncology 92: 360-370, 2017.

19. Bombonati A and Sgroi DC: The molecular pathology of breast cancer progression. J Pathol 223: 307-317, 2011.

20. Osako T, Iwase T, Ushijima M, Horii R, Fukami Y, Kimura K, Matsuura $\mathrm{M}$ and Akiyama F: Incidence and prediction of invasive disease and nodal metastasis in preoperatively diagnosed ductal carcinoma in situ. Cancer Sci 105: 576-582, 2014.

21. Chan MY and Lim S: Predictors of invasive breast cancer in ductal carcinoma in situ initially diagnosed by core biopsy. Asian J Surg 33: 76-82, 2010.

22. Kim J, Han W, Lee JW, You JM, Shin HC, Ahn SK, Moon DG, Cho N, Moon WK, Park IA and Noh DY: Factors associated with upstaging from ductal carcinoma in situ following core needle biopsy to invasive cancer in subsequent surgical excision. Breast 21: 641-645, 2012.

23. Yoshikawa M: Sentinel node biopsy in patients diagnosed with ductal carcinoma in situ preoperatively. Japanese Soc Sentinel Node Navigation Surg 18: 45, 2016.

24. Kodahl AR, Lyng MB, Binder H, Cold S, Gravgaard K, Knoop AS and Ditzel HJ: Novel circulating microRNA signature as a potential non-invasive multi-marker test in ER-positive early-stage breast cancer: A case control study. Mol Oncol 8: 874-883, 2014.

25. Huang L, Li F, Deng P and Hu C: MicroRNA-223 promotes tumor progression in lung cancer A549 cells via activation of the $\mathrm{NF}-\kappa \mathrm{B}$ signaling pathway. Oncol Res 24: 405-413, 2016.

26. Li X, Zhang Y, Zhang H, Liu X, Gong T, Li M, Sun L, Ji G, Shi Y, Han Z, et al: miRNA-223 promotes gastric cancer invasion and metastasis by targeting tumor suppressor EPB41L3. Mol Cancer Res 9: 824-833, 2011.

27. Pinatel EM, Orso F, Penna E, Cimino D, Elia AR, Circosta P, Dentelli P, Brizzi MF, Provero P and Tavern D: miR-223 is a coordinator of breast cancer progression as reveraled by bioinformatics predictions. PLoS One 9: e84859, 2014.

28. Sun X, Li Y, Zheng M, Zuo W and Zheng W: MicroRNA-223 increases the sensitivity of triple-negative breast cancer stem cells to TRAIL-induced apoptosis by targeting HAX-1. PLoS One 11: e0162754, 2016. 